\title{
On the relationship between force reduction, loading rate and energy absorption in athletics tracks
}

\author{
Luca Andena ${ }^{1,3}$, Antonio Ciancio ${ }^{1}$, Francesco Briatico-Vangosa ${ }^{1}$, Stefano Mariani ${ }^{2,3}$, Andrea \\ Pavan 1 \\ ${ }^{1}$ Dipartimento di Chimica, Materiali e Ingegneria Chimica, Politecnico di Milano, Italy \\ ${ }^{2}$ Dipartimento di Ingegneria Civile e Ambientale, Politecnico di Milano, Italy \\ ${ }^{3} E^{4}$ Sport Lab, Politecnico di Milano, Italy \\ Corresponding author: \\ Luca Andena, Assistant Professor, Polymer Engineering Lab, Dipartimento di Chimica, Materiali \\ e Ingegneria Chimica "G. Natta", Politecnico di Milano, Piazza Leonardo da Vinci, 32 - 20133, \\ Milano (Italy). \\ E-mail luca.andena@polimi.it; web http://www.chem.polimi.it/polyenglab/ \\ tel +3902 2399 3289; fax +3902 23993280
}

\section{Keywords}

Sports surfaces; running tracks; numerical modelling; force reduction; energy absorption;

loading rate

\section{Abstract}

In the present work finite element simulations of typical sports surfaces were performed to evaluate parameters, such as the loading rate and the energy absorbed by the surface, in relation to its characteristics (surface structure and material properties). Hence, possible relations between these quantities and the standard parameters used to characterize the shock absorbing characteristics of the athletics track (in particular its Force Reduction) were investigated.

The samples selected for this study were two common athletics tracks and a sheet of natural rubber. They were first characterized by quasi-static compression tests; their mechanical properties were extrapolated to the strain rate of interest and their dependence on the level of deformation was modelled with hyperelastic constitutive equations.

Numerical simulations were carried out for varying sample thicknesses, in order to understand the influence of track's geometry on Force Reduction, loading rate and stored energy. A very 
good correlation was found between Force Reduction and the other relevant parameters, with the exception of the loading rate at the beginning of the impact.

\section{Introduction}

Sports surfaces play a fundamental role in athletes' activities as proven by the fact that surfaces with specific properties are required for different sports. Surfaces in use today are both natural and artificial. The latter type has been undergoing a considerable development for several decades [1-2], for different reasons: the need to improve their functionality and durability even under adverse weather conditions, the opportunity to decrease production and maintenance costs, the possibility to improve the athlete's performance while ensuring her/his safety. As for the performance, the successful contribution of new track surfaces to the establishing of new world records during running events cannot be undervalued. An important result attained with the newly developed tracks is the change in magnitude and direction of the loads transmitted back from the ground to the human locomotive apparatus; to this regard several works in the literature highlighted the relevant role played by the running track properties, and in particular their stiffness $[1,3-5,6]$. This brought an associated change in the typology and frequency of injuries, as already recognized long ago (e.g. in [6]). More recent studies investigated surfaceathlete's body interactions [2,7] and particularly the energy exchange aspects, making use of numerical analysis techniques such as finite element (FE) modelling [8].

When considering the methods used to characterize the mechanical behaviour of a sports surface it is important to distinguish between subject tests and material tests [2]. The standard tests prescribed by IAAF (International Association of Athletics Federations) to approve running tracks belong to the second category [9]. Among this second kind of tests great importance is given to the so-called 'Force Reduction' test [10], which is performed by using an apparatus called Artificial Athlete (Berlin). The latter is composed of a $20 \mathrm{~kg}$ dropping a mass falling from a height of $55 \mathrm{~mm}$ onto a rigid plate placed on top of a spring; the load is then transmitted to a load cell connected to a base plate (a steel disc, $70 \mathrm{~mm}$ in diameter), which ultimately rests on the track sample to be tested. The Force Reduction $(F R)$ is obtained by comparing the maximum 
value, $F_{\max }$, of the vertical force recorded by the load cell during the test with a corresponding reference value, $F_{r}$, obtained by performing the same experiment on a surface without cushioning properties (e.g. concrete), according to:

$$
F R=\left(1-\frac{F_{\max }}{F_{r}}\right) \times 100
$$

The FR parameter is considered to be an index of the shock absorption ability of the track. It turned out to be dependent not only on the intrinsic properties of the track material, as suggested by Durà et al. [11], but also on extrinsic features, such as the geometrical structure and the thickness of the surface, as demonstrated by the experimental work of Benanti et al. [12]. To investigate the net effect of the material properties on the shock absorption characteristics of running tracks, Andena et al. [13-14] subsequently assessed and took into account the hyperelastic behaviour of some of the materials studied in [12] and developed a predictive FE model of the FR test.

The aim of the present work is to investigate the influence of some parameters, such as the loading rate and the maximum absorbed energy, by exploiting the proposed model, thus getting further insight into the impact behaviour of a given surface.

The loading rate [15-16] is commonly related to the first peak of the force-time curve resulting from subject tests conducted with a force platform. Although so far overlooked by IAAF, this parameter seems to be of a crucial importance for the athlete's comfort and well-being. In particular, high loading rates seem to sensibly reduce bone fatigue resistance and easily result in fractures, whereas no significant effect of the maximum vertical load (used to define $F R$ ) seems to have been detected in this kind of test. Loading rate is also known to be more sensitive than the maximum force to changes in cushioning [19]. As for the impact energy, its absorption and restitution by a surface are of paramount importance for optimizing cushioning and performance, respectively [8]. 
The overall aim of the present investigation is to obtain a more complete assessment of all influential characteristics of a track and to determine the possible relations existing between these parameters of potential biomechanical interest and FR.

\section{Materials and methods}

\section{$2.1 \quad$ Materials}

Five different materials were considered in this study: two of them ( $A$ and $B$ ) are real tracks, while the third one (NR) is a 'dummy' track, already studied previously and identified with the same code as used in [12-14]; the remaining two are fictitious materials used in some of the numerical simulations to explore a broader range of material properties. More specifically:

- A: a $16 \mathrm{~mm}$ thick in-situ resin-bound rubber crumb running track

- B: a $13.5 \mathrm{~mm}$ thick prefabricated running track, with a top finishing layer and a bottom base layer having a rectangular honeycomb structure

- NR: a $8 \mathrm{~mm}$ thick sheet of natural rubber

- 1 and 2: two fictitious hyperelastic materials with the same density as NR but higher stiffness

Higher thicknesses in the experiments were achieved by stacking multiple track layers. Samples were freely stacked as previous works [12] already demonstrated that the outcome of the $F R$ tests is not affected by the presence of glue, meaning that friction between the layers is enough to suppress any significant amount of relative sliding. 


\section{$2.2 \quad$ FR tests}

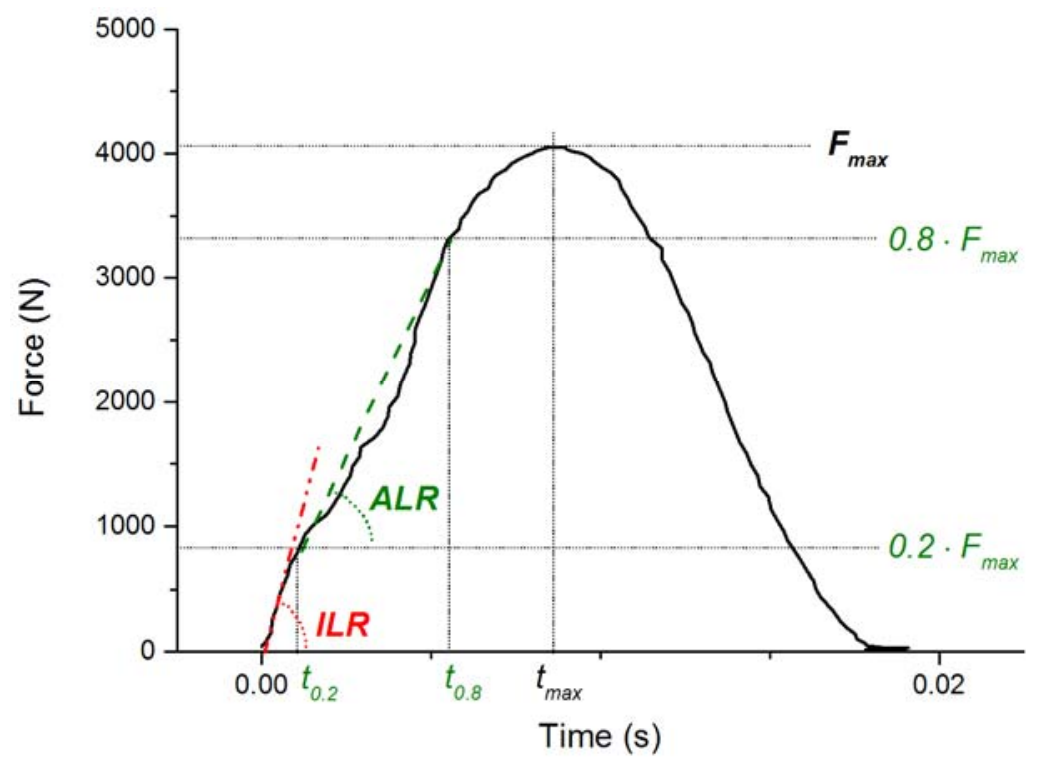

Figure 1. Definition of characteristic parameters on a typical force vs. time curve obtained in a FR test ( $F_{\max }$ : force peak; ILR: Initial Loading Rate; ALR: Average Loading Rate). For the definitions of $t_{0.2}$ and $t_{0.8}$ : see text.

Load-time data (such as those shown in Figure 1) measured during $F R$ tests performed with an artificial athlete apparatus were taken from [12]. Namely, the parameters considered in the analysis of the experiments were:

- $F R$, the Force Reduction, related to the force peak $F_{\max }$, as per the definition in equation (1). The value was calculated after the experimental force-time curve was processed using a ninth order Butterworth filter with a cut-off frequency of $120 \mathrm{~Hz}$, as required by IAAF standards [10]

- ILR, the Initial Loading Rate, calculated from the best linear fit of the initial part (the first $1.5-2 \mathrm{~ms}$ ) of the load vs. time curve, in which the slope is almost constant and unaffected by subsequent load oscillations

- $A L R$, the Average Loading Rate in the time interval between $t_{0.2}$ and $t_{0.8}$, calculated as the average slope in the range between $20 \%$ and $80 \%$ of $F_{\max }$, according to: 


$$
A L R=\frac{0.8 \cdot F_{\max }-0.2 \cdot F_{\max }}{t_{0.8}-t_{0.2}}
$$

$t_{0.2}$ and $t_{0.8}$ being the instants when the force $F$ reaches $20 \%$ and $80 \%$ of $F_{\max }$ for the first time, respectively.

The definitions given for ILR and $A L R$ are consistent with those adopted by several other authors (see [15] for a review).

\subsection{Compression tests}

Stress-stretch data obtained from quasi-static uniaxial compression tests at varying loading rates (in the range 0.006-0.6 $\mathrm{s}^{-1}$ ) were taken from [13-14]. A new extrapolation scheme (described later) was devised to obtain relevant data for the investigated materials at an effective rate of $60 \mathrm{~s}^{-1}$, previously [13] determined to be consistent with $F R$ testing.

\section{$2.4 \quad$ Numerical simulations}

FE simulations of the FR tests were carried out using the commercial FE code Abaqus (Simulia) [17]. Details on the characteristics of the FE model used were thoroughly reported in [13]; for each track sample, a two-dimensional (2D) space discretization was adopted exploiting the axi-symmetry in the solution governed by the propagation of stress waves in the through-thethickness direction, as induced by the mass drop. The degree of mesh refinement was checked to lead to objective, i.e. mesh-independent, results for all the track performance indices here investigated. As for the Artificial Athlete apparatus, steel parts were considered as rigid bodies with the exception of the spring and load cell, modelled as elastic springs having stiffness and mass corresponding to the instrument manufacturer's specifications. To avoid convergence issues, unilateral contact between the different bodies was enforced using a penalty method.

The simulations pertained to both the materials investigated experimentally $(A, B$ and $N R)$ and the two fictitious materials ( 1 and 2 ) having arbitrarily assigned properties, all listed above.

The load vs. time curves output obtained from the simulations were analysed in terms of the same parameters determined from the experiments $(F R, I L R, A L R)$. In addition, from the output of the numerical model also the strain energy stored in each material element and hence the 
whole strain energy, $U$, elastically stored in the material could be determined. To reduce the influence of load oscillations on the evaluation of the energy storage capability, the $U$ vs. time curves were filtered with the same ninth order Butterworth filter used on the $F$ vs. time curves for the determination of $F R$. An example is shown in Figure 2.

Accordingly, two additional parameters, which characterise $U$ vs. time curves, were considered:

- $U_{\max }$, the Maximum Storage Energy, corresponding to the peak in the stored energy vs. time curve of the $F R$ test

- USR, the average Energy Storage Rate, calculated from the storage energy vs. time curve in a similar way as $A L R$ is calculated from load vs. time curves:

$$
U S R=\frac{0.8 \cdot U_{\max }-0.2 \cdot U_{\max }}{t_{0.8}^{\prime}-t_{0.2}^{\prime}}
$$

where $t^{\prime} 0.2$ and $t^{\prime} 0.8$ are the instants when the stored energy $U$ reaches $20 \%$ and $80 \%$ of $U_{\max }$ for the first time, respectively.

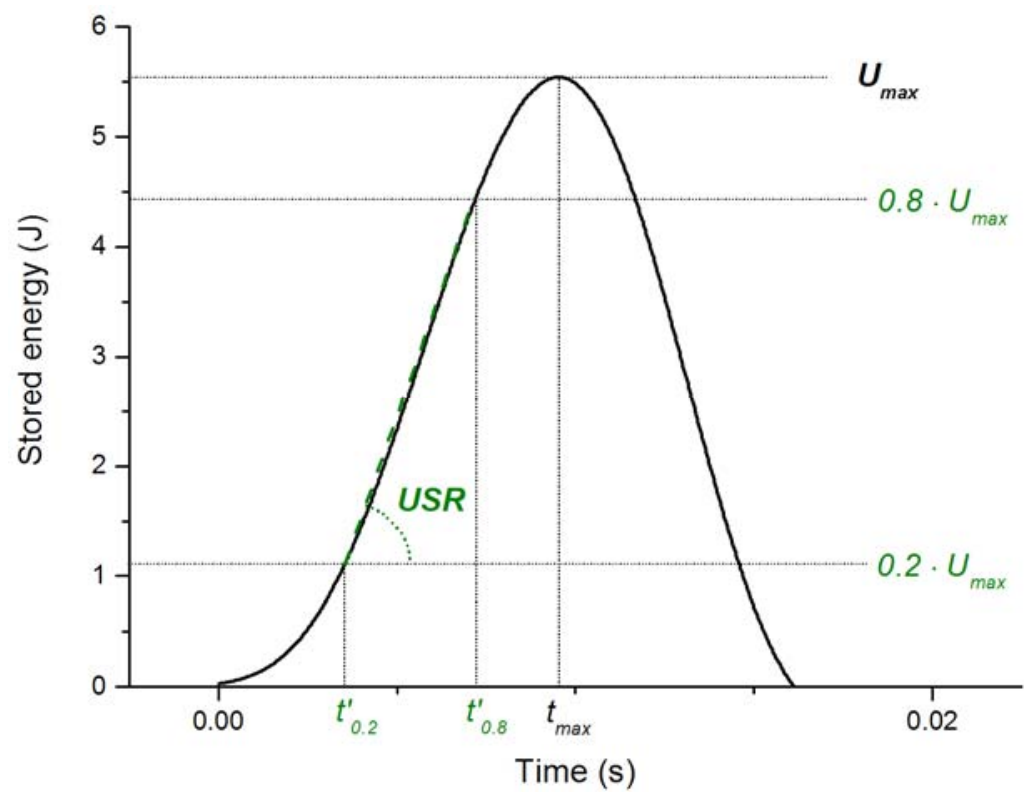

Figure 2. Definition of characteristic parameters on a typical stored energy, $U$, vs. time curve obtained from the numerical simulations of a FR test $\left(U_{\max }\right.$ : Maximum Storage Energy; USR: average Energy Storage Rate) 


\subsection{Constitutive modelling}

Following the results found in [13], the Mooney-Rivlin (MR) hyperelastic equation [18] was chosen to model the mechanical behaviour of the investigated materials (assumed as incompressible) within the FE code. Under uniaxial loading conditions, the unidimensional relationship between the tensile stress $\sigma$ and the stretch ratio $\lambda=L / L_{0}$, with $L$ and $L_{0}$ being the current and initial lengths of the material specimen, reads:

$$
\sigma=2\left(C_{10}+\frac{C_{01}}{\lambda}\right)\left(\lambda-\frac{1}{\lambda^{2}}\right)
$$

In compression, the stretch ratio $\lambda$ becomes less than unity and so $\sigma$ is negative. In equation (4), $C_{10}$ and $C_{01}$ are material parameters which, within the small strain limit, can be related to the effective elastic modulus, $E$, through:

$$
2\left(C_{10}+C_{01}\right)=\frac{E}{3}
$$

For each material in [13-14], data at a given constant stretch rate were fitted with equation (4); the resulting values of the coefficients $C_{10}$ and $C_{01}$ were then plotted against the applied stretch rate and extrapolated to $60 \mathrm{~s}^{-1}$. The fit outcome turned out to be rather insensitive to the actual values of the two MR parameters and affected by a certain degree of scatter.

To improve the result, a different approach was then adopted. The sought stress-stretch curves at $60 \mathrm{~s}^{-1}$ were obtained by linearly extrapolating the stress vs. log(stretch rate) data for each level of stretch, in 0.01 intervals. The resulting curves were then fitted with equation (4).

The outcome is shown in Figure 3 for the three materials under investigation. Their properties, together with those of the two fictitious materials later used in the numerical simulations, are listed in Table 1. 


$\begin{array}{ccccc}\text { Material } & \mathbf{C}_{10}(\mathbf{M P a}) & \mathbf{C}_{01}(\mathbf{M P a}) & \mathbf{E}(\mathbf{M P a}) & \text { Density } \mathbf{( k g / \mathbf { m } ^ { 3 } )} \\ \mathbf{A} & 0.21 \pm 0.03 & 0.19 \pm 0.02 & 2.4 & 640 \\ \mathbf{B} & 0.47 \pm 0.04 & 0.09 \pm 0.02 & 3.6 & 930 \\ \text { NR } & 1.05 \pm 0.13 & 0.85 \pm 0.08 & 11.4 & 1390 \\ \mathbf{1} & 5 & 0.85 & 35.1 & 1390 \\ \mathbf{2} & 10 & 0.85 & 65.1 & 1390\end{array}$

Table 1. Mooney-Rivlin coefficients, elastic modulus and density of all materials.

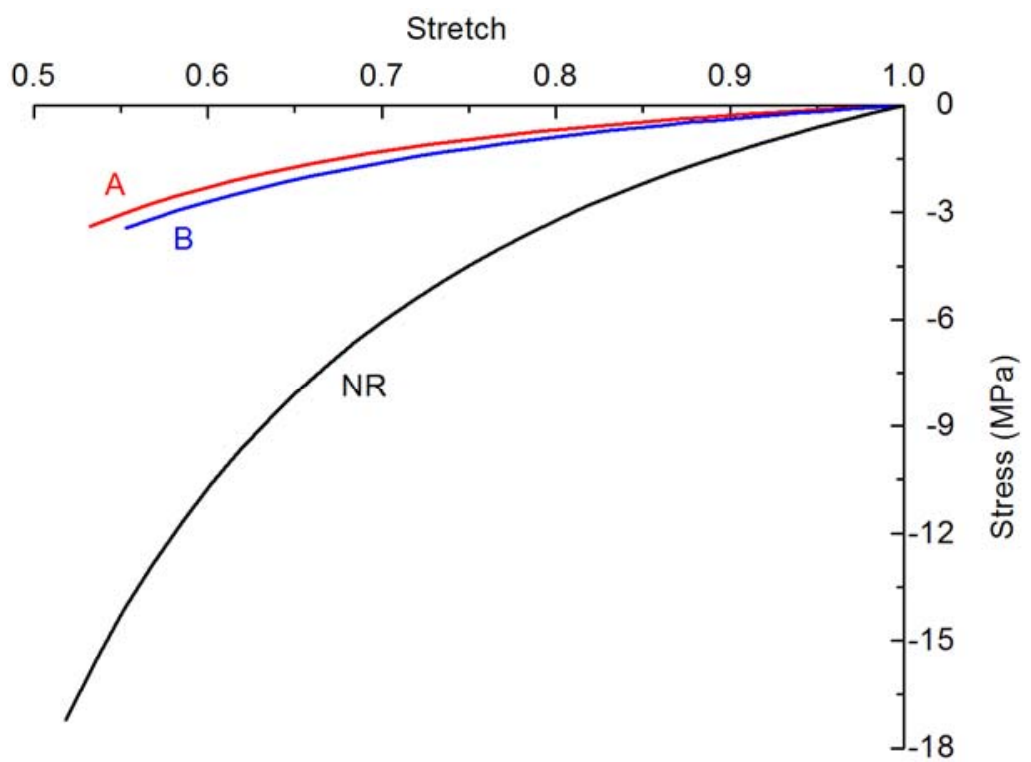

Figure 3. Stress vs. stretch curves at $60 \mathrm{~s}^{-1}$ for the three materials $A, B$ and NR, as obtained from extrapolation of uniaxial compression data at lower rates

\section{Results and discussion}

\subsection{Force reduction}

The typical outcome of a numerical simulation performed using the aforementioned FE model is shown in Figure 4 together with relevant experimental data for the case of material $A$, as an example. The graphs shows the experimental results and relevant simulation of a FR tests on a 4-layer assembly, in which (as previously reported in [12-13]) additional oscillations in the load curve appear, with respect to the typical outcome of a test on a single track layer. It is clear that the model provides an accurate description of the dynamic response of the material being tested. In particular, it captures quite well the maximum load value, which is used in the FR determination. 


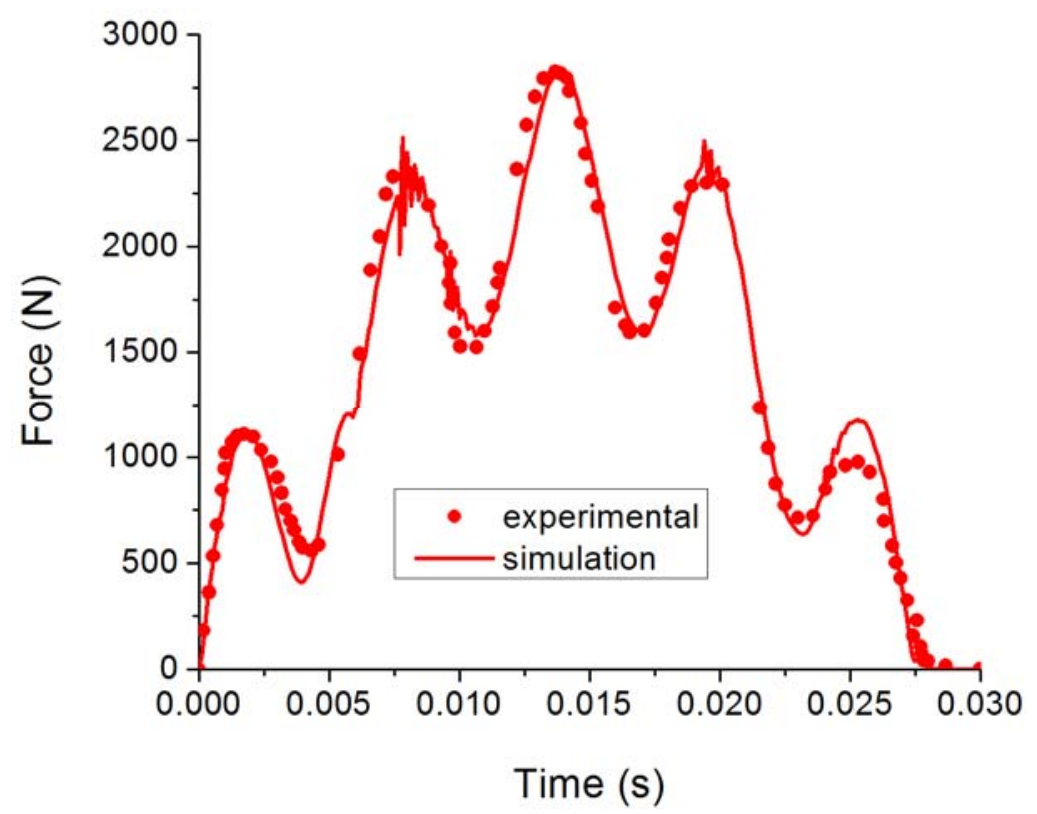

Figure 4. Comparison between experimental and numerical force vs. time data from a FR test on material A with $64 \mathrm{~mm}$ thickness (4 layers)

Figure 5 shows $F R$ values obtained from numerical simulations performed on the three materials $(A, B, N R)$ at varying thicknesses, up to $120 \mathrm{~mm}$, compared with relevant experimental data. The agreement is very good on the whole range of thicknesses for materials $A$ and NR. In the case of material B, the numerical model underestimates $F R$ more and more as thickness increases; this is most probably due to the inherent honeycomb structure of the prefabricated track $B$, a feature that at present is not considered in the axisymmetric $2 \mathrm{D} \mathrm{FE}$ model. While material properties were determined on single track layers under quasi-static constant stretching-rate conditions, the effective response of a stack of honeycomb layers under dynamic (high-rate) conditions is likely to differ because of the complex deformation of the base layer of the prefabricated track. 


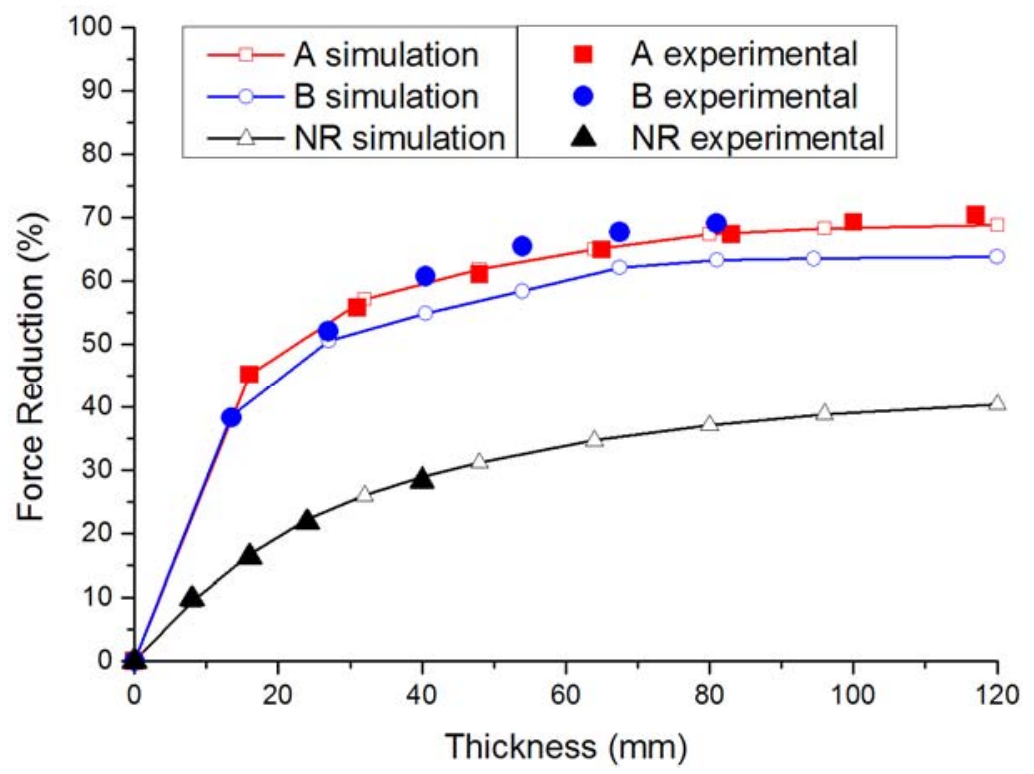

Figure 5. Comparison between experimental and simulated Force Reduction data at varying thicknesses for materials $A, B$ and NR

\subsection{Loading rate}

Figure 6 displays values of $A L R$ (as previously defined) vs. $F R$ in a $F R$ range between $20 \%$ and $70 \%$ for a combination of materials and thicknesses. The graph includes FR and ALR data both obtained experimentally and calculated from relevant numerical simulations. A strong correlation between the two quantities, $A L R$ and $F R$, can be observed, as could be expected in view of the assumed elastic behaviour of the track during the FR test. Indeed, the change in momentum $M$ calculated by integration of the force-time curve has approximately the same value of about $41.6 \mathrm{Ns}$ for all the tests - that is, about twice the value of the momentum possessed by the dropping mass upon impact. This is consistent with an almost elastic impact of the dropping mass, which bounces back from the track.

As a first approximation $M$ could be related to $F_{\max }$ as follows:

$$
M=2 \int_{0}^{t_{\text {max }}} F d t=p F_{\text {max }} t_{\text {max }}
$$


indicating with $p$ a shape factor characterising the shape of the force-time curve ( $p=1$ for a linear load-time curve).

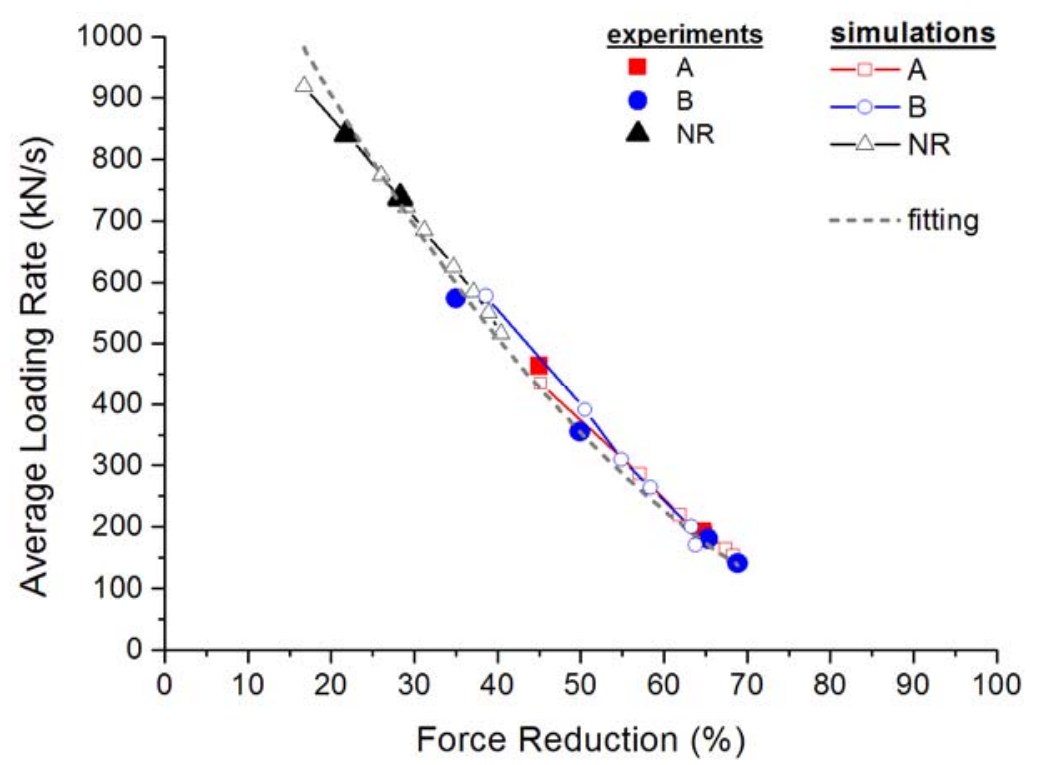

Figure 6. Average Loading Rate vs. Force Reduction for materials A, B and NR; the dashed line is a fit of all data according to equation (8).

On the other hand, $A L R$ can be related to $F_{\max }$ and $t_{\max }$ by:

$$
A L R=q \frac{F_{\max }}{t_{\max }}
$$

indicating with $q$ another load curve shape factor accounting for the definition of ALR over a range of $F$ values limited between $20 \%$ and $80 \%$ of $F_{\max }$ ( $q$ would be 1 if $A L R$ was calculated on the whole load curve up to $\left.F_{\max }\right)$.

By combining equations (1), (6) and (7) one can obtain:

$$
A L R=p q \frac{F_{r}^{2}}{M}\left(1-\frac{F R}{100}\right)^{2}=K\left(1-\frac{F R}{100}\right)^{2}
$$

If $K$ is assumed to be constant, reflecting the fact that the load curves for the different materials had a similar shape, $A L R$ is predicted to decrease quadratically with increasing $F R$. 
The fitting of equation (8) to all the data points (both experimental and numerical) reported in Figure 6 yields the grey dashed line, with a best fit value $K=1430 \mathrm{kN} / \mathrm{s}$.

Referring to this apparently 'universal' relationship between $A L R$ and $F R$ one can note that the $F R$ range of $35-50 \%$ established by IAAF standards as acceptable $F R$ values thus corresponds to an $A L R$ interval of about $350-600 \mathrm{kN} / \mathrm{s}$.

Yet, the loading rate actually experienced by the artificial athlete during the very first instants of the impact, represented by ILR, is usually higher, as it can be observed for example in Figures 1 and 4. Values of ILR were then evaluated for all cases examined above, both experimental and numerically simulated; they are shown in Figure 7 as a function of sample thickness.

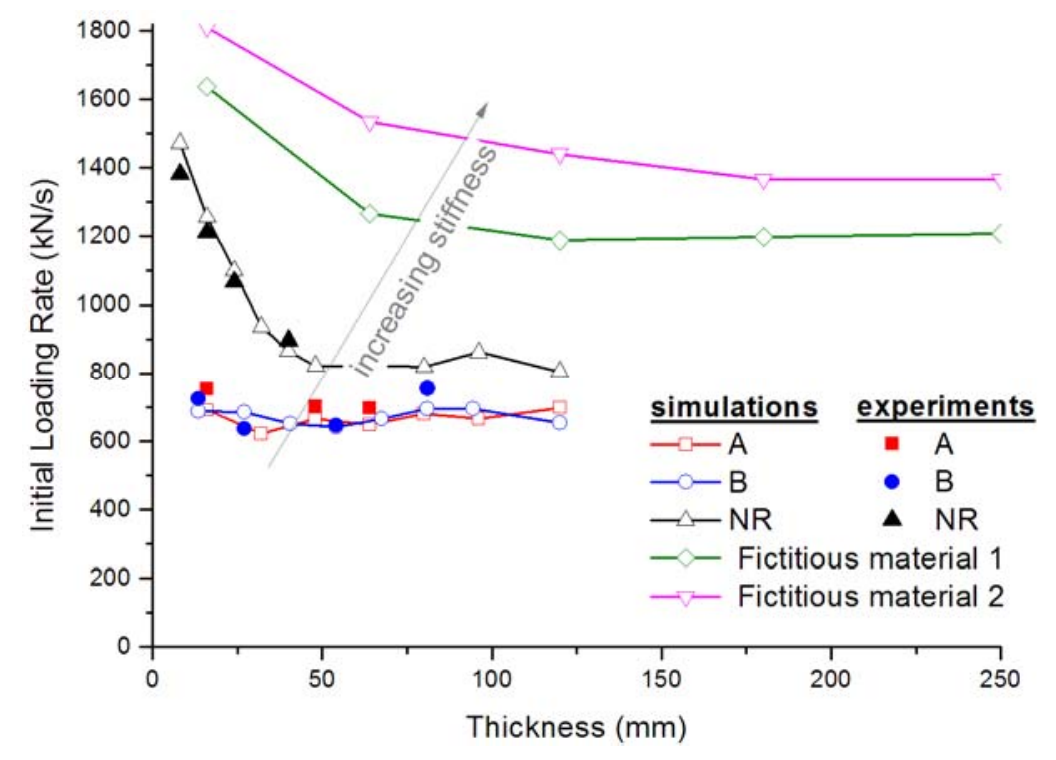

Figure 7. Initial Loading Rate, ILR, vs. sample thickness for all materials; the increasing stiffness indication (arrow) refers to the effective elastic moduli listed in Table 1.

It is noteworthy the good agreement of the ILR values obtained by numerical analysis with the experimental data for materials $A, B$ and NR. For the two real tracks (materials $A$ and $B$ ), the value of ILR is fairly constant, about $700 \mathrm{kN} / \mathrm{s}$, over the whole thickness range explored. The 'dummy' track material, NR, by contrast, displays significantly higher values and a strongly 
decreasing trend of ILR with increasing sample thickness up to about $50 \mathrm{~mm}$, above which numerical simulations predict ILR to level off at a value slightly above $800 \mathrm{kN} / \mathrm{s}$. Besides having a slightly higher density, NR differs from the athletics tracks $A$ and $B$ in its significantly larger stiffness (see Table 1). This finding suggests that a material with higher stiffness requires a larger thickness to reach its asymptotic behaviour (see also Figure 5) and its asymptotic ILR value remains higher than that of softer materials. To confirm this observation, additional simulations were run using the two fictitious materials, 1 and 2, which were assigned the same $C_{01}$ coefficient as for NR but larger $C_{10}$ values: they correspond to effective moduli values about 3 and 6 times larger than for NR. The simulations confirm the previous experimental observation: the relevant plateau is located at higher ILR values and is reached at higher thickness. Last, it is worth observing that by contrast with $A L R$, IRL results are not expected to and do not directly correlate with $F R$.

\subsection{Energy Absorption}

A general trend can be observed in the Maximum Storage Energy $\left(U_{\max }\right)$ : it increases as the track's stiffness decreases or its thickness increases. This is consistent with the fact that the total energy is composed of contributions associated with the deformation of the track and with other elements of the testing apparatus (e.g the spring): a more compliant surface absorbs a larger part of the available impact energy. Obviously the spring's presence also affects the measurements of the other (load-related) parameters such as FR, ALR and ILR, by modulating the load transfer from the mass to the track surface. This issue is inherent to the Artificial Athlete setup and becomes no doubt more important when evaluating energy absorption because of the aforementioned partition on the total impact energy. The question could be addressed by a specific investigation of the influence of the spring's stiffness, but this goes beyond the scope of the present work.

It is interesting to verify if a correlation between $U_{\max }$ and $F R$ does exist (Figure 8). Indeed, as in the case of the $A L R$ vs. FR diagram (Figure 6), this is the case: all the data for the different 
materials collapses onto a single curve. This result depends on the combined effects of the track's stiffness and thickness, as proven in [12].

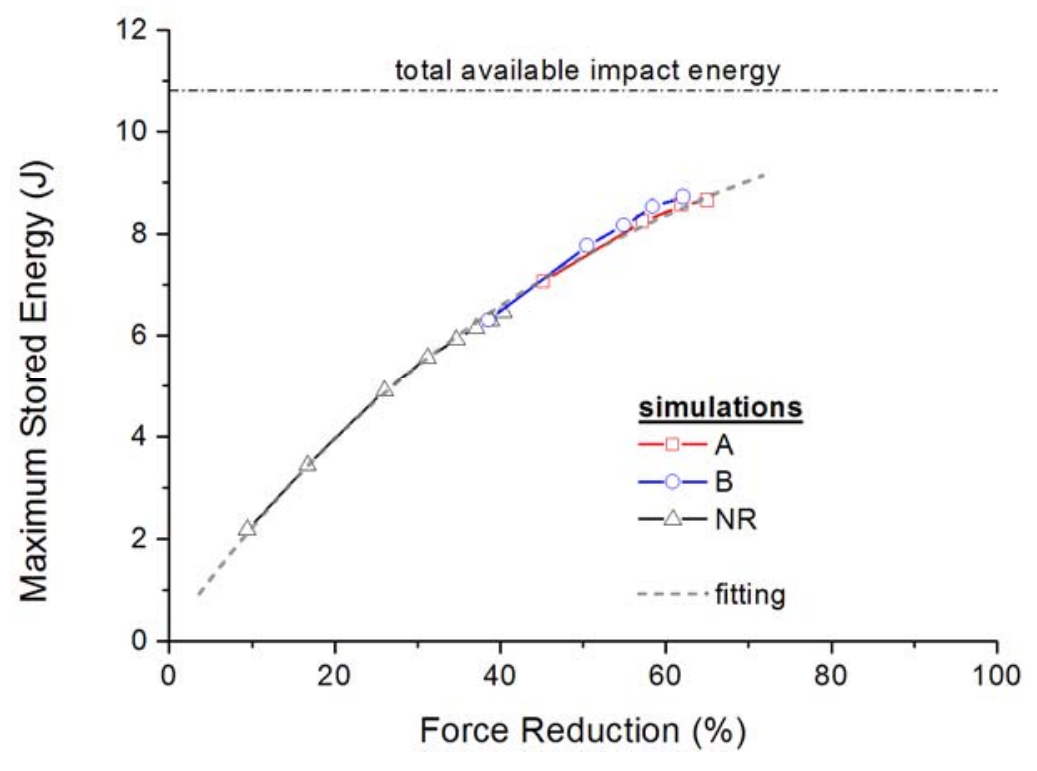

Figure 8. Maximum Stored Energy vs. Force Reduction for materials A, B, NR; the dashed line is a fit of all data according to equation (9)

An empirical exponential function:

$$
U_{\max }=U_{0}-U_{1} e^{-\frac{F R}{F R_{U}}}
$$

can satisfactorily fit the $U_{\max }$ vs. $F R$ data with the following values of the three parameters: $U_{0}=12.2 \mathrm{~J} ; U_{1}=12.0 \mathrm{~J} ; F R U=52 \%$.

A very interesting result is found for the rate at which the energy is stored in the material, USR. Given the almost symmetrical shape of the force vs. time curves (see e.g. Figure 1), this quantity is also representative of the resilience, i.e. the energy return characteristics, of the surfaces (in the vertical direction). Figure 9 shows USR data for different combinations of materials and thicknesses, again as a function of $F R$. Once more, all data points collapse onto a single curve, confirming that the $F R$ value of a given track is a direct indication also of its energy 
absorption and energy return characteristics, in terms of both $U_{\max }$ and USR. The curve shows a broad maximum of about $1000 \mathrm{~J} / \mathrm{s}$, which is around the higher end of the $F R$ approval range established by IAAF (35-50\%). This maximum arises from the competition between opposite effects. As FR increases starting from very low values (corresponding to very stiff and/or thin surfaces), the stored energy increases and also USR grows; at the same time, however, the impact duration increases as well. For very high values of $F R$ (i.e. very compliant and/or thick surfaces) this effect ends up by dominating and the rate of energy absorption decreases.

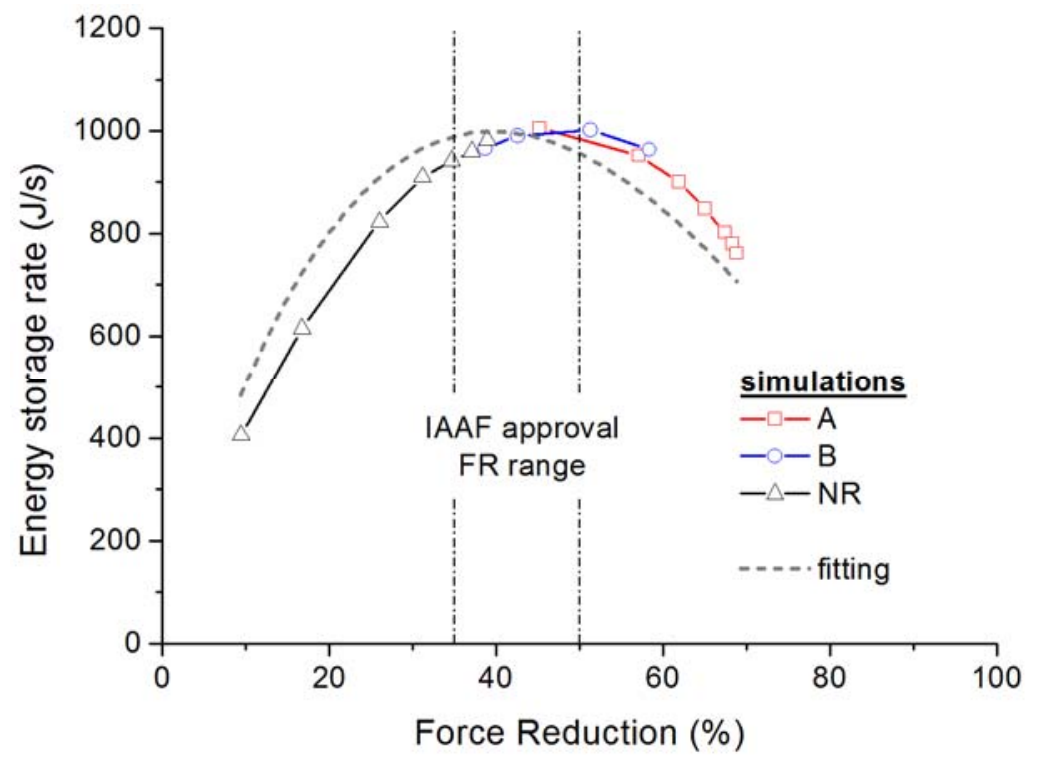

Figure 9. Energy Storage Rate (USR) vs. Force Reduction (FR) for materials A, B and NR; the dashed line is a fit of equation (9) to all data

Following the same path used in the analysis of the loading rate results, a prediction for the expected dependence of USR on FR can be obtained. In particular USR can be related to the peak stored energy, $U_{\max }$, and the peak time, $t_{\max }\left({ }^{*}\right)$, via:

$$
U S R=q^{\prime} \frac{U_{\max }}{t_{\max }}
$$

(") Strictly, the instant when USR reaches its maximum may not coincide with $t_{\max }$, the time of the maximum load, but for the sake of simplicity we neglect here the possible difference. 
in which $q^{\prime}$ is a shape factor, having the same role of the coefficient $q$ in equation (7).

Combining equation (10) with equations (1), (6) and (9) one can obtain:

$$
U S R=\frac{p q^{\prime}}{2 M} F_{r}\left(1-\frac{F R}{100}\right) U_{\max }=K^{\prime}\left(1-\frac{F R}{100}\right)\left(U_{0}-U_{1} e^{-\frac{F R}{F R_{U}}}\right)
$$

The data shown in Figure 9 were fitted by equation (11), yielding a best fit value $K^{\prime}=2.55 \mathrm{~s}^{-1}$. The quality of the agreement is good, although this simplified analytical prediction gives a curve (dashed line in Figure 9) which is slightly shifted towards lower FR values with respect to the experimental data. Nevertheless, equation (11) highlights the complex influence of $F R$ on the energy storage rate parameter and its trend with a broad maximum which, perhaps not incidentally, falls in the acceptable $F R$ range established by the IAAF standard.

The above results and considerations strongly depend on the actual characteristics of the Artificial Athlete (in particular the spring's stiffness): any change in the test setup would significantly alter the amount of impact energy absorbed and also the relevant rate. Yet, for a given test setup, similar trends are expected when considering tracks having different characteristics.

\section{Conclusions}

In the present work a recently developed [13] numerical FE model able to simulate Force Reduction $(F R)$ testing was used to investigate how sport surfaces having different geometrical characteristics and mechanical properties respond in terms of several parameters of biomechanical interest.

The approach adopted for the identification of the material parameters from laboratory tests was slightly different from the one used previously. A good agreement between FR experimental data and numerical predictions was assessed for various combinations of material stiffness and 
thicknesses, with some minor differences reported only in the case of a prefabricated track with honeycomb base layer and high thickness.

Most of the parameters investigated (Average Loading Rate - ALR, Maximum Storage Energy $U_{\max }$ and Energy Storage Rate - USR) turned out to bear a direct correlation with FR and a set of empirical relationships (supported by mechanical analysis) has been made available to well describe these correlations.

In particular, it was found that the rate at which the track material absorbs the impact energy, USR, shows a maximum just in the FR range that is prescribed by IAAF for their approval. Thus $F R$ is proven to be a simple, yet scientifically sound parameter, able to synthetically characterise the shock absorption characteristics of running tracks.

One reservation concerns the Initial Loading Rate (ILR) experienced at the beginning of the impact, which seems to be not simply related to FR. This is most likely due to the dynamic (i.e. inertial) effects that are significantly present in the initial part of the test and that cannot be captured by a single parameter. ILR turned out to be almost independent of $F R$ for actual track materials over a broad range of thicknesses. This means that even if track characteristics (stiffness and thickness) are adjusted to modify shock absorption, the observed variation in $F R$ will not translate into a different ILR; this fact may justify discrepancies between laboratory and subject tests that were previously reported on various tracks [2].

\section{Acknowledgements}

This work was partially supported by Fondazione Cariplo, Milano (Italy) under the project "Safer Helmets". 


\section{References}

1. MacMahon TA, Greene PR The influence of track compliance on running. J Biomech 1979; 12:893-904.

2. Nigg BM, Yeadon MR. Biomechanical aspects of playing surfaces. J Sports Sci 1987; $5: 117-145$.

3. Farley $\mathrm{C}$, Houdijk $\mathrm{H}$, Strien $\mathrm{CV}$, et al. Mechanism of leg stiffness adjustment for hopping on surfaces of different stiffnesses. J Appl Phys 1998; 85:1044-1055.

4. Kerdok AE, Biewener AA, McMahon TA, et al. Energetics and mechanics of human running on surfaces of different stiffnesses. J Appl Phys 2002; 92:469-478.

5. Brughelli M, Cronin J. A review of research on the mechanical stiffness in running and jumping: methodology and implications. Scand J Med Sci Sports 2008; 18:417-426.

6. James SL, Bates BT, Osterning LR. Injuries to runners. Am J Sports Med 1978; 6:4050.

7. Carré MJ, James DM, Haake SJ. A hybrid method for assessing the performance of sports surfaces during ball impacts. Proc. Inst. Mech. Eng. L J. Mater. Des. Appl. 2006; 220:31-39.

8. Baroud G, Nigg BM, Stefanyshyn DJ. Energy storage and return in sport surfaces. Sports Eng 1999; 2:173-180.

9. IAAF. Track and Field Facilities Manual, 2008.

10. EN 14808:2005. Surfaces for sports areas - Determination of shock absorption.

11. Durà JV, Garcia AC, Solaz J. Testing shock absorbing materials: the application of viscoelastic linear model. Sports Eng 2002; 5:9-14.

12. Benanti M, Andena L, Briatico-Vangosa $F$, et al. Characterization of the viscoelastic behavior of athletics track surfaces in relation to their "force reduction" property. Polym Test 2013; 32:52-59.

13. Andena L, Briatico-Vangosa F, Ciancio A, et al. Modeling of shock absorption in athletics track surfaces. Sports Eng 2015; 18:1-10. 
14. Andena L, Briatico-Vangosa F, Cazzoni E, et al. A finite element model for the prediction of Force Reduction of athletics tracks. Proc Eng 2014; 72:847-852.

15. Zadpor AA, Nikooyan AA. The relationship between lower-extremity stress fractures and the ground reaction force: a systematic review. Clin Biomech 2011; 26:23-28.

16. Schaffler MB, Radin EL, Burr DB. Mechanical and morphological effects of strain rate on fatigue of compact bone. Bone 1989; 10:207-214.

17. Dassault Systèmes. Abaqus 6.13 User Manual, 2013.

18. Macosko CW. Rheology. Principles, measurements and applications. $1^{\text {st }}$ ed. New York: Wiley-VCH, 1994, p.43.

19. Dixon SJ. Use of pressure insoles to compare in-shoe loading for modern running shoes. Ergonomics 2008; 51:1503-14. 